\title{
Assessment of commutation failure in HVDC systems considering spatial-temporal discreteness of AC system faults
}

\author{
Huanhuan YANG ${ }^{1}$, Zexiang CAI ${ }^{1}$, Xiaohua LI $^{1}$, Chaoyun YU ${ }^{1}$
}

Abstract This paper presents a novel commutation failure (CF) assessment method considering the influences of voltage magnitude drop, phase shift, and spatial-temporal discreteness of AC system faults. The commutating voltage-time area is employed to analyze the spatial-temporal discreteness of AC system faults causing $\mathrm{CF}$ in high-voltage direct current systems, and the influences of fault position and fault time on $\mathrm{CF}$ are revealed. Based on this, a novel $\mathrm{CF}$ criterion is proposed, further considering the influence of voltage phase shift and the spatial-temporal discreteness. Then this research develops a new $\mathrm{CF}$ assessment method, which does not rely on electromagnetic transient simulations. A real case from the China Southern Power Grid is used to verify the practicability of the proposed method by comparing with simulation results obtained using PSCAD/EMTDC.

Keywords Commutation failure, Spatial-temporal discreteness, Phase shift, Assessment method

CrossCheck date: 2 November 2017

Received: 10 December 2016/Accepted: 2 November 2017/ Published online: 5 February 2018

(C) The Author(s) 2018. This article is an open access publication

Zexiang CAI

epzxcai@scut.edu.cn

Huanhuan YANG

yhappy1208@foxmail.com

Xiaohua LI

eplxh@scut.edu.cn

Chaoyun YU

yucy_scut@163.com

1 School of Electric Power, South China University of Technology, Guangzhou 510640, China

\section{Introduction}

Commutation failure (CF) is an adverse and frequent dynamic event in high-voltage direct current (HVDC) systems based on line-commutated converter technology. It is most often caused by AC system faults in the receivingend grid [1]. CF causes a drastic increase in DC currents at the DC side, which poses a serious threat to converter equipment, and causes temporary interruption of transmitted active power, leading to instability issues on the AC side [2]. Therefore, it is crucial to establish a systematic assessment method for CF caused by AC system faults, which can assist $\mathrm{CF}$ prevention and contingency response.

Over decades, many efforts have been made to assess the immunity of HVDC systems against CF caused by AC system faults. Simulation-based assessment methods are common approaches to assess CF. References [3, 4] use methods based on electromechanical transient simulation to assess CF. Although their computational efficiency can be ensured, the assessment results are not accurate enough due to shortcomings of the quasi-steady-state HVDC model. Methods based on electromagnetic transient simulation are believed to be more accurate [1], considering that the commutation process of HVDC converter valves is modeled in detail and a CF can be identified by checking behaviors of valve switching. Nevertheless, this method inevitably leads to heavy workload and long computation time for large-scale AC/DC hybrid power systems, which cannot meet application requirements of power system planning and operation.

Considering disadvantages of the simulation-based assessment method, another idea is to develop analytical assessment methods which are convenient for sensitivity analysis, screening studies, and so on. Earlier approaches to 
assess the vulnerability of the converter to $\mathrm{CF}$ used the maximum permissible balanced voltage drop on the converter's AC bus [1]. If the voltage dropped by more than this amount, CF was presumed to occur. Reference [5] adopted 0.9 p.u. as the critical voltage (namely 0.1 p.u. voltage magnitude drop). However, [6] shows that the above voltage-drop criterion sometimes gives results that do not match those obtained through detailed electromagnetic transient simulation. For some AC system faults, CF occurs when the $\mathrm{AC}$ bus voltage drops by more than a critical value, while $\mathrm{CF}$ is avoided for other $\mathrm{AC}$ system faults which cause the same voltage drop. The voltage phase shift, which has an important influence on CF [7], should also be considered. That is to say, conditions for the occurrence of CF depend on the fault location and the fault time (i.e. the point on the waveform at which a fault occurs) [8-10], so the occurrence of $\mathrm{CF}$ is related to the spatial-temporal discreteness of $\mathrm{AC}$ system faults.

In [11, 12], a commutation failure immunity index (CFII) is proposed to assess the immunity of an HVDC converter to CF. However, to obtain the CFII, electromagnetic transient simulations are required for calculating the smallest possible fault inductance that does not result in a CF. Reference [13] provided an analytical expression of a CFII based on the simulation result that the CFII is insensitive to the fault time under a three-phase inductive fault condition. However, in contrast to CFIIs for threephase faults, CFIIs for single-phase faults are closely related to the fault time [12].

In order to guarantee a successful commutation in thyristor valves, a certain minimum commutating voltagetime area is required, and this is provided by a minimum extinction angle $\gamma_{\text {min }}$. The cause of CFs is that the actual extinction angle is less than $\gamma_{\min }$ [14-16]. Reference [17] introduced an expression of the extinction angle, which is a function of the commutating voltage, the DC current, the ignition advance angle, and the phase shift. However, the influence of the phase shift on the extinction angle was derived from empirical or heuristic reasoning (simply subtracting the phase shift from the extinction angle). Besides, the effects of spatial-temporal discreteness of AC system faults on CF should also be taken into consideration.

This research aims to develop an efficient approach for $\mathrm{CF}$ assessment based on an accurate $\mathrm{CF}$ criterion. The spatial-temporal discreteness of AC system faults causing $\mathrm{CF}$ is analyzed using the commutating voltage-time area, through which the impact mechanism of the fault position and the fault time on $\mathrm{CF}$ is revealed. Then a novel analytical CF criterion based on the minimum extinction angle is derived directly from the equivalent circuit of the commutation process, considering both the magnitude drop and the phase shift of the post-fault commutating voltage. On this basis, an approach to $\mathrm{CF}$ assessment is proposed. Instead of employing electromagnetic transient simulations, the proposed assessment method can be accomplished through a short-circuit computing program, which provides an efficient and practical way to assess $\mathrm{CF}$ in large-scale power systems.

\section{Spatial-temporal discreteness of $\mathrm{CF}$}

\subsection{Commutating voltage-time area}

Figure 1 is the schematic diagram of a 6-pulse bridge converter. In this figure, $I_{\mathrm{d}}$ is the DC current, $L_{\mathrm{c}}$ is the equivalent commutating inductance per phase, $e_{a}, e_{b}$, and $e_{c}$ are the AC phase voltages, and $\mathrm{V}_{1}$ to $\mathrm{V}_{6}$ are the converter valves numbered sequentially.

Taking the commutation from $\mathrm{V}_{4}$ to $\mathrm{V}_{6}$ as an example, Fig. $2 \mathrm{a}$ and Fig. $2 \mathrm{~b}$ show the commutation process under normal conditions and under an AC system fault condition, respectively. In Fig. $2, e_{c b}$ is the pre-fault commutating voltage, $e_{c b}^{\prime}$ is the post-fault commutating voltage, $\Delta \varphi$ is the phase shift, $t_{1}$ is the commutation starting time, $t_{2}$ is the commutation ending time, $\beta$ is the ignition advance angle, $\gamma$ is the extinction angle, $\mu$ is the overlap angle, $P_{6}$ is the trigger pulse of $\mathrm{V}_{6}, i_{4}$ and $i_{6}$ are valve currents of $\mathrm{V}_{4}$ and $\mathrm{V}_{6}$, respectively, $S_{\mathrm{cr}}$ is the commutating voltage-time area required for a successful commutation process, and $S_{\mathrm{f}}$ is the maximum available commutating voltage-time area under the AC fault condition. Note that a variable with the subscript " $\mathrm{f}$ " denotes a corresponding variable in fault conditions.

Assuming that the DC current is kept constant by a DC current controller and a large smoothing reactor in normal operating conditions, then $S_{\mathrm{cr}}$ is a constant given by:

$S_{\mathrm{cr}}=2 L_{\mathrm{c}} I_{\mathrm{d}}$

Due to the magnitude drop and phase shift forward of the post-fault commutating voltage, the available

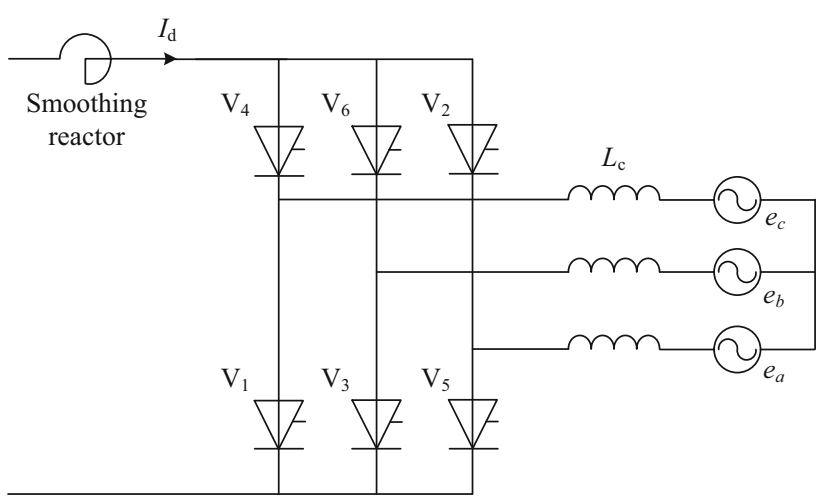

Fig. 1 Schematic diagram of 6-pulse bridge converter 

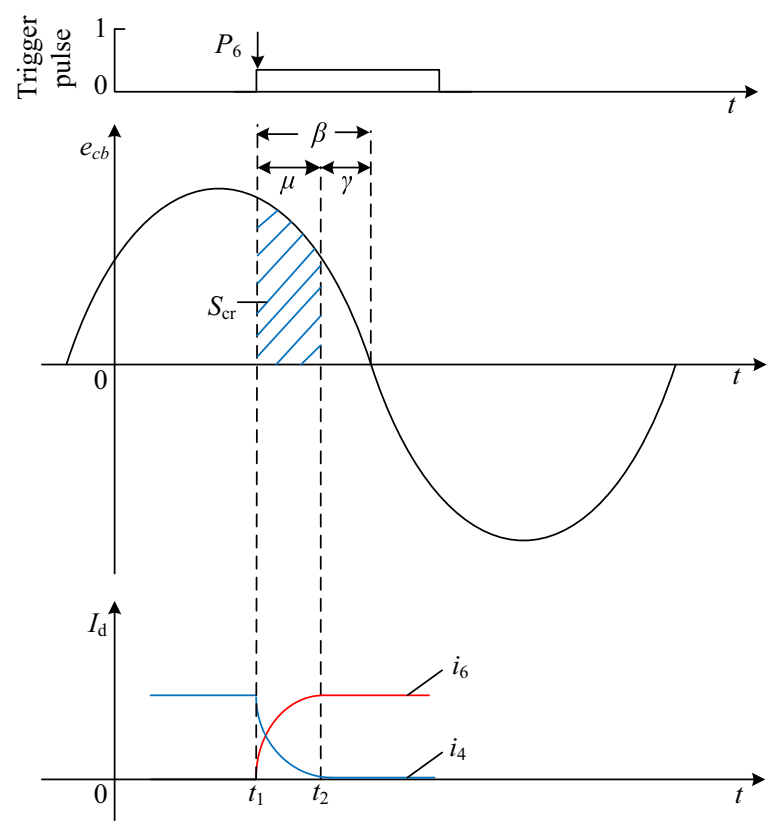

(a) Under normal condition
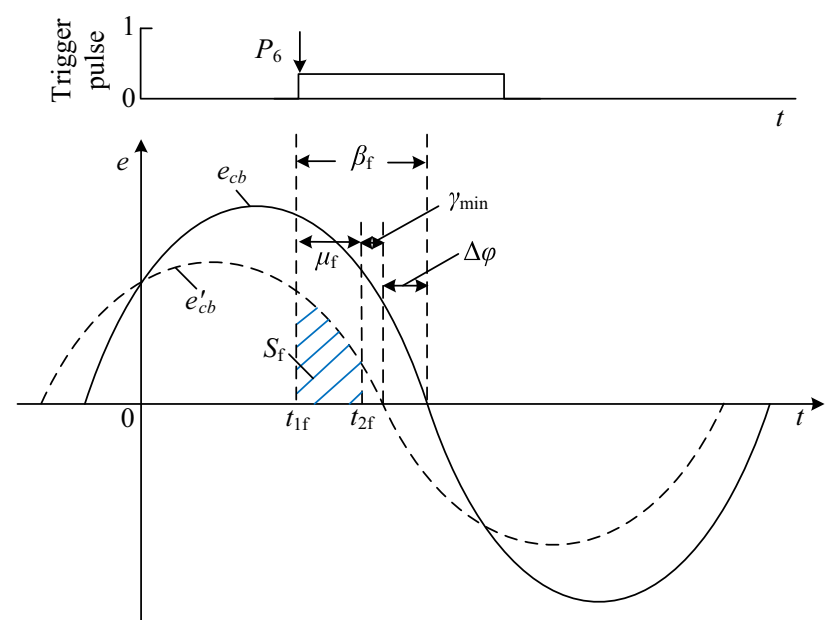

(b) Under AC system fault condition

Fig. 2 Commutation process from $\mathrm{V}_{4}$ to $\mathrm{V}_{6}$

commutating voltage-time area will decrease. If the $\mathrm{AC}$ system fault occurs before the original firing instant of the valve, the HVDC controller will trigger the valve at the fault instant in order to increase the commutating voltagetime area $S_{\mathrm{f}}$. If the AC system fault occurs after the original firing instant, there is no time for HVDC controller to adjust the firing instant, and according to the natural response characteristics of valves, the commutation process will lengthen to attempt to increase the commutating voltage-time area $S_{\mathrm{f}}$.

If $S_{\mathrm{f}}<S_{\mathrm{cr}}$, the commutation process is unable to complete before $t_{2 \mathrm{f}}$, and a CF occurs because the extinction angle is less than $\gamma_{\min }$. If $S_{\mathrm{f}}>S_{\mathrm{cr}}$, the commutation process is completed before $t_{2 \mathrm{f}}$, and a CF is avoided. If $S_{\mathrm{f}}=S_{\mathrm{cr}}$, the converter is in the critical $\mathrm{CF}$ state, which means that $\gamma=\gamma_{\text {min }}$. Thus $S_{\mathrm{f}}$ is used as an index to quantify the ability of a converter to prevent $\mathrm{CF}$.

\subsection{Temporal discreteness of AC faults causing CF}

The dependence of CF on fault time is referred to as the temporal discreteness of AC faults. The commutating voltage-time area is employed to perform a detailed analysis of temporal discreteness.

Continuing to take the commutation from $\mathrm{V}_{4}$ to $\mathrm{V}_{6}$ as an example, it is noted that $e_{c b}^{\prime}$ is related to the fault position, the fault type and the fault level, without being affected by the fault time. Since the phase difference between $e_{c b}$ and $t_{\mathrm{p}}^{\prime}$ has no effect on the analysis of temporal discreteness, $e_{c b}^{\prime}$ is assumed to be in phase with $e_{c b}$ for simplicity. Figure 3 shows the influence of the fault time on the commutating voltage-time area. In this figure, $t_{\mathrm{I}}$ and $t_{\mathrm{II}}$ are example fault times, $t_{\mathrm{p}}$ is the firing instant, $t_{\mathrm{p}}^{\prime}$ is the forward firing instant, $t_{\gamma}$ is the time corresponding to $\gamma_{\min }$, and $\Delta S_{\mathrm{I}}$ and $\Delta S_{\mathrm{II}}$ are the increased commutating voltage-time areas corresponding to faults at $t_{\mathrm{I}}$ and $t_{\mathrm{II}}$ respectively. These two cases are now considered.

\subsubsection{Fault occurs before firing instant}

If a fault occurs at the firing instant $t_{\mathrm{p}}$, the maximum available commutating voltage-time area, $S_{\mathrm{f} 0}$, is

$S_{\mathrm{f} 0}=\int_{t_{\mathrm{p}}}^{t_{y}} e_{c b}^{\prime} \mathrm{d} t$

If a fault occurs before $t_{\mathrm{p}}\left(\mathrm{e} . \mathrm{g}\right.$., at $\left.t_{\mathrm{I}}\right)$, the firing instant of $P_{6}$ is shifted forward to $t_{\mathrm{p}}^{\prime}\left(t_{\mathrm{p}}^{\prime}>t_{\mathrm{I}}\right)$ by HVDC control. Note that $t_{\mathrm{p}}^{\prime}>t_{\mathrm{I}}$, due to the response time of HVDC control. Under this condition, the maximum available commutating voltage-time area, $S_{\mathrm{fI}}$, is

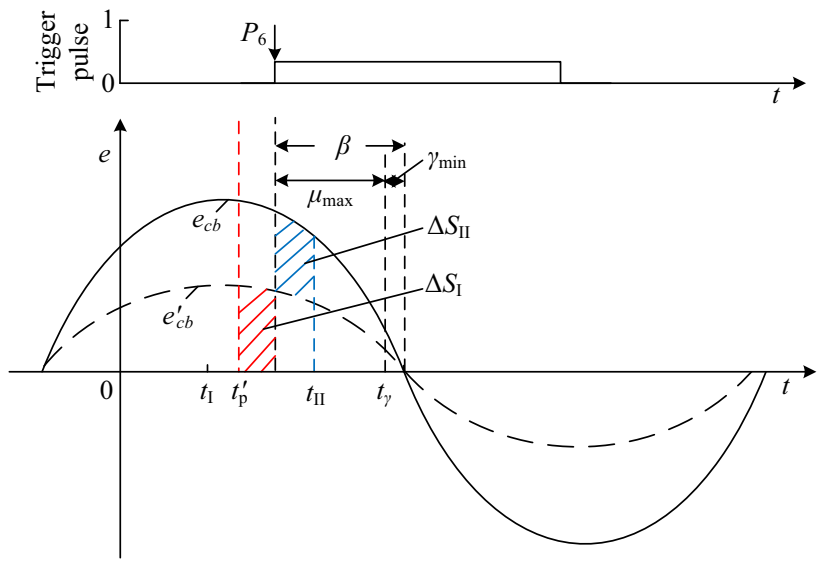

Fig. 3 Influence of fault time on commutating voltage-time area 
$S_{\mathrm{fI}}=\int_{t_{\mathrm{p}}^{\prime}}^{t_{\mathrm{p}}} e_{c b}^{\prime} \mathrm{d} t+\int_{t_{\mathrm{p}}}^{t_{\gamma}} e_{c b}^{\prime} \mathrm{d} t=\Delta S_{\mathrm{I}}+S_{\mathrm{fo}}$

From (2) and (3), it can be seen that if a fault occurs before the firing instant, HVDC control shifts the firing instant forward by increasing $\beta$, and thus enlarges $S_{\mathrm{fo}}$ by $\Delta S_{\mathrm{I}}>0$ shown in Fig. 2, which is beneficial to commutation.

\subsubsection{Fault occurs after firing instant}

If a fault occurs after $t_{\mathrm{p}}$ (e.g., at $t_{\mathrm{II}}$ ), HVDC control has no effect on the commutating voltage-time area. Under this condition, the influence of the fault time on the commutating voltage-time area, $S_{\mathrm{f}}$, depends on the lag of $t_{\mathrm{II}}$ behind $t_{\mathrm{p}}$.

$$
\begin{aligned}
S_{\mathrm{fII}} & =\int_{t_{\mathrm{p}}}^{t_{\mathrm{II}}} e_{c b} \mathrm{~d} t+\int_{t_{\mathrm{II}}}^{t_{\gamma}} e_{c b}^{\prime} \mathrm{d} t \\
& =\int_{t_{\mathrm{p}}}^{t_{\mathrm{II}}}\left(e_{c b}-e_{c b}^{\prime}\right) \mathrm{d} t+\int_{t_{\mathrm{p}}}^{t_{\gamma}} e_{c b}^{\prime} \mathrm{d} t=\Delta S_{\mathrm{II}}+S_{\mathrm{f} 0}
\end{aligned}
$$

Equations (2) and (4) show that if a fault occurs after the firing instant, $S_{\mathrm{f}}$ is larger than $S_{\mathrm{f} 0}$, and the increased area is $\Delta S_{\text {II }}>0$, which is also beneficial to commutation.

\subsubsection{Most adverse fault time for commutation}

From the above, it is observed that both $S_{\mathrm{fI}}$ and $S_{\mathrm{fII}}$ are larger than $S_{\mathrm{fo}}$. Therefore the most adverse fault time for a valve's commutation process is at the firing instant, under the assumption that the fault is applied on the phase which is connected to the anode of the valve.

\subsection{Spatial discreteness of $\mathrm{AC}$ faults causing $\mathrm{CF}$}

The occurrence of $\mathrm{CF}$ is a function of the steady-state voltage phase difference and the electrical distance between the fault bus and the commutation bus. This phenomenon is referred to as the spatial discreteness of AC faults causing CF. In order to simplify the analysis, without loss of generality, a three-phase fault is taken as an example.

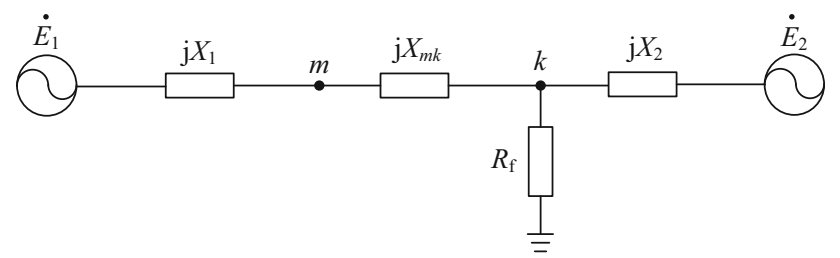

Fig. 4 Equivalent circuit for a three-phase fault with resistance $R_{\mathrm{f}}$ at bus $k$
Figure 4 shows the equivalent circuit, where a threephase fault with fault resistance $R_{\mathrm{f}}$ is applied at bus $k$. Bus $m$ is the commutating bus, $\dot{E}_{1}$ and $\dot{E}_{2}$ are the equivalent sources, and $X_{1}, X_{2}$ and $X_{m k}$ are the equivalent reactances.

From Fig. 4 , the post-fault line voltage of bus $k, \dot{U}_{k A B}$, is

$\dot{U}_{k A B}=\dot{E}_{e q A B}-\sqrt{3} \frac{X_{e q}}{R_{f}+\mathrm{j} X_{e q}} \dot{E}_{e q A B} \mathrm{e}^{120^{\circ}}$

where

$\dot{E}_{e q A B}=\frac{X_{2} \dot{E}_{1}+\left(X_{1}+X_{m k}\right) \dot{E}_{2}}{X_{1}+X_{2}+X_{m k}}$

$X_{e q}=\frac{X_{2}\left(X_{1}+X_{m k}\right)}{X_{1}+X_{2}+X_{m k}}$

The post-fault line voltage of bus $m, \dot{U}_{m A B}$, is

$\dot{U}_{m A B}=\frac{X_{m k}}{X_{m k}+X_{1}} \dot{E}_{1 A B}+\frac{X_{1}}{X_{m k}+X_{1}} \dot{U}_{k A B}$

From (6), it is observed that $\dot{U}_{m A B}$ is the linear combination of $\dot{E}_{1 A B}$ and $\dot{U}_{k A B}$. According to (5) and (6), the vector graphs of $\dot{U}_{k A B}$ and $\dot{U}_{m A B}$ are plotted in Fig. 5, where dashed lines denote the trajectories of $\dot{U}_{k A B}$ and $\dot{U}_{m A B}$ as $R_{\mathrm{f}}$ varies between $\infty$ and zero.

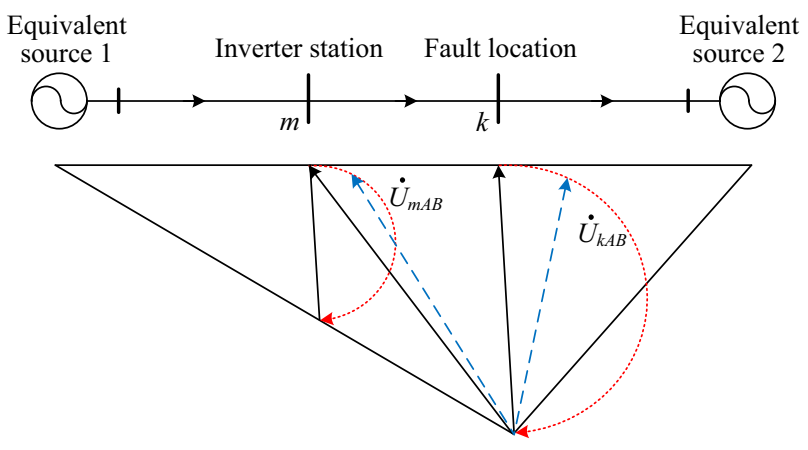

(a) $\dot{U}_{k A B}$ lags behind $\dot{U}_{m A B}$

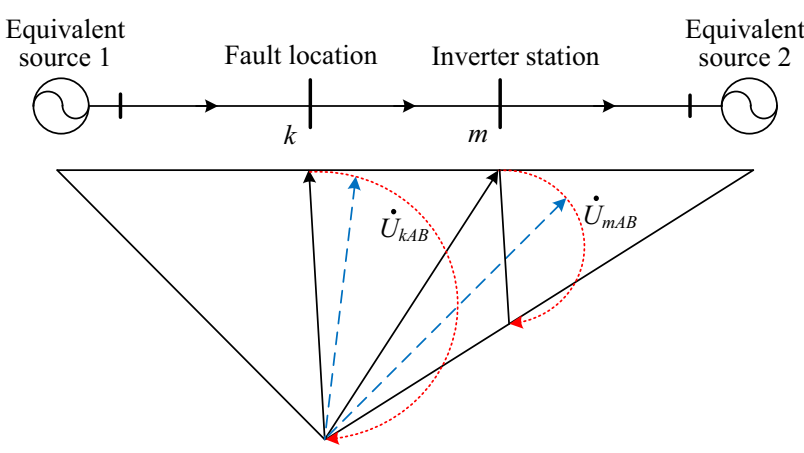

(b) $\dot{U}_{k A B}$ leads $\dot{U}_{m A B}$

Fig. 5 Voltage vector graphs of bus $k$ and bus $m$ 
The influence of the steady-state voltage phase difference between the fault bus and the commutation bus on $\mathrm{CF}$ is analyzed as follows.

\subsubsection{Pre-fault phase of $\dot{U}_{k A B}$ lags behind that of $\dot{U}_{m A B}$}

Assuming that the active power in Fig. 4 flows from $\dot{E}_{1}$ to $\dot{E}_{2}$, if $k$ is closer to $\dot{E}_{2}$ than $m$, the pre-fault phase of $\dot{U}_{k A B}$ lags behind that of $\dot{U}_{m A B}$. From Fig. 5a, the magnitude of $\dot{U}_{m A B}$ decreases for a fault, and the phase of $\dot{U}_{m A B}$ is shifted forward when $R_{\mathrm{f}}$ is small, which are both adverse to commutation.

\subsubsection{Pre-fault phase of $\dot{U}_{k A B}$ leads that of $\dot{U}_{m A B}$}

Continuing to assume that the active power in Fig. 4 flows from $\dot{E}_{1}$ to $\dot{E}_{2}$, if bus $m$ is closer to $\dot{E}_{2}$ than bus $k$, the pre-fault phase of $\dot{U}_{k A B}$ leads that of $\dot{U}_{m A B}$. From Fig. 5b, the magnitude of $\dot{U}_{m A B}$ decreases for a fault, which is adverse to commutation. However, the phase of $\dot{U}_{m A B}$ is shifted backward regardless of the value of $R_{\mathrm{f}}$, which is beneficial to commutation.

Based on the above analysis, if the commutating voltage magnitude drops are same, the phase-leading voltage at the fault position is more beneficial to commutation than the phase-lagging voltage.

As for the influence on $\mathrm{CF}$ of the electrical distance between the fault bus and the commutation bus, from Fig. 5a and Fig. 5b, the less the electrical proximity, the more severe the voltage drop of $\dot{U}_{m A B}$, and the commutation bus is the most adverse fault position for commutation.

The analysis results show that the magnitude and phase of the post-fault commutating voltage are influenced by the steady-state voltage phase difference and the electrical distance between the fault bus and the commutating bus, which embodies the spatial discreteness of AC faults. A fault occurring in closer electrical proximity and with a bigger phase lag is more adverse to commutation.

\section{Assessment criterion for $\mathrm{CF}$ considering magnitude drop and phase shift of commutating voltage}

\subsection{Theoretical derivation of assessment criterion}

Under normal conditions, according to the equivalent circuit when the current is commutating from $\mathrm{V}_{4}$ to $\mathrm{V}_{6}$, $e_{a b}=e_{b}-e_{a}=L_{\mathrm{c}} \frac{\mathrm{d} i_{\mathrm{k}}}{\mathrm{d} t}-L_{\mathrm{c}} \frac{\mathrm{d}\left(I_{\mathrm{d} 0}-i_{\mathrm{k}}\right)}{\mathrm{d} t}$

where $i_{\mathrm{k}}$ is the circular current in the commutation circuit; $I_{\mathrm{d} 0}$ is the pre-fault DC current. This formula can be rewritten as:

$2 \frac{\mathrm{d} i_{\mathrm{k}}}{\mathrm{d} t}=\sqrt{2} E_{a c 0} \sin (\omega t)$

where $E_{a c 0}$ is the magnitude of the pre-fault commutating voltage. Then,

$i_{\mathrm{k}}=-\frac{\sqrt{2} E_{a c 0}}{2 \omega L_{\mathrm{c}}} \cos (\omega t)+C_{0}$

where $C_{0}$ is the integration constant under normal conditions.

When $\omega t=\pi-\beta_{0}, i_{\mathrm{k}}=0$, then $C_{0}$ is calculated as:

$C_{0}=-\frac{\sqrt{2} E_{a c 0}}{2 \omega L_{\mathrm{c}}} \cos \left(\beta_{0}\right)$

When $\omega t=\pi-\gamma_{0}, i_{\mathrm{k}}=I_{\mathrm{d} 0}$, then the pre-fault DC current $I_{\mathrm{d} 0}$ is:

$I_{\mathrm{d} 0}=\frac{\sqrt{2} E_{a c 0}}{2 \omega L_{\mathrm{c}}}\left(\cos \left(\gamma_{0}\right)-\cos \left(\beta_{0}\right)\right)$

When a fault occurs in the AC system, and does not cause a $\mathrm{CF}$,

$2 \frac{\mathrm{d} i_{\mathrm{k}}}{\mathrm{d} t}=\sqrt{2} E_{a c} \sin (\omega t+\Delta \phi)$

$i_{\mathrm{k}}=-\frac{\sqrt{2} E_{a c}}{2 \omega L_{c}} \cos (\omega t+\Delta \phi)+C_{1}$

where $E_{a c}$ is the magnitude of the post-fault commutating voltage; $C_{1}$ is the integration constant under fault conditions.

Because it takes a certain amount of time for HVDC control to respond after a fault, it is reasonable to assume that $\beta_{0}$ is a constant during this time interval.

Similarly to the calculation of $I_{\mathrm{d} 0}$, the post-fault DC current $I_{\mathrm{d} 1}$ without $\mathrm{CF}$ is expressed as:

$I_{\mathrm{d} 1}=\frac{\sqrt{2} E_{a c}}{2 \omega L_{c}}\left(\cos \left(\gamma_{\mathrm{f}}-\Delta \phi\right)-\cos \left(\beta_{0}-\Delta \phi\right)\right)$

where $\gamma_{\mathrm{f}}$ is the post-fault extinction angle.

Since there is no CF, the DC system is treated as a constant current source, which means that $I_{\mathrm{d} 0}=I_{\mathrm{d} 1}$. Under this condition, $\gamma_{\mathrm{f}} \in\left[\gamma_{\min }, \gamma_{0}\right]$. Generally, in real HVDC projects, $\gamma_{\min }$ is about $7^{\circ}$, and $\gamma_{0}$ is about $18^{\circ}$, and if a $\mathrm{CF}$ is not caused, $\Delta \varphi$ is relatively small for any type of AC fault. Therefore, it is reasonable to assume that the value of $\gamma_{\mathrm{f}}-\Delta \varphi$ is within the range of $0^{\circ}-90^{\circ}$.

Combining (11) and (14) gives an expression for the post-fault extinction angle: 
$\left\{\begin{array}{l}\gamma_{\mathrm{f}}=\arccos (M)+\Delta \varphi \\ M=\cos \left(\gamma_{0}-\Delta \varphi\right) \frac{E_{a c 0}}{E_{a c}}+\cos \left(\beta_{0}-\Delta \varphi\right)\end{array}\right.$

\subsection{Discussion on developed assessment criterion}

The derivation of the post-fault extinction angle is based on the conditions that there is no CF and $I_{\mathrm{d} 0}=I_{\mathrm{d} 1}$. If the DC system suffers a CF following a fault, there exists a significant change in the DC current.

If the fault is severe, there is a large change in the commutating voltage. In this case, according to the concept of commutating voltage-time area, the commutation process fails to complete before the commutating voltage reverses, thus leading to a $\mathrm{CF}$. Consequently, the calculation method of (11) is inapplicable for the derivation of (14). Abnormal values of $\gamma_{\mathrm{f}}$ may be obtained using (15) due to a large $E_{a c 0}$ compared to $E_{a c}$ as well as $|\Delta \varphi|$ : when $M>1, \arccos (M)$ in (15) has no real solution; when $M \approx 1, \arccos (M) \approx 0$, and $\gamma_{\mathrm{f}} \approx \Delta \varphi$, which means that $\gamma_{\mathrm{f}}$ is less than zero or $\gamma_{\mathrm{f}}$ is relatively large.

If the fault is minor, the commutating voltage has a small change. In this case, a CF happens when a converter valve that has turned off conducts again automatically when a forward voltage is applied to it, because the voltage blocking capability is not recovered during the reverse voltage. Thus (14) is still applicable. Because $E_{a c 0}$ is not much larger than $E_{a c}$ and $|\Delta \varphi|$ in (15) is relatively small, $M<1$. However, $M$ is reduced by the assumption of no change in the DC current, $I_{\mathrm{d} 0}=I_{\mathrm{d} 1}$, leading to a small increase in $\gamma_{\mathrm{f}}$ compared to $\gamma$ that considers the change in $I_{\mathrm{d}}$. Under critical CF conditions, it is likely that the assessment criterion will incorrectly predict a $\mathrm{CF}$ when in fact commutation succeeds.

According to the above analysis, if $\gamma_{\mathrm{f}}$ is within the range from 0 to $\gamma_{\min }$, or $\gamma_{\mathrm{f}}$ has an abnormal value, it identifies that the DC system suffers a CF; if $\gamma_{\mathrm{f}}$ is within the range from $\gamma_{\min }$ to $\gamma_{0}$, it indicates that the DC system survives a CF, although with a possible false prediction of $\mathrm{CF}$ under critical CF conditions. Therefore, the proposed criterion for identifying $\mathrm{CF}$ is effective and is not affected by a change in the DC current.

As analyzed in Section 2, the onset of CF has the characteristics of temporal discreteness, and the most adverse fault time for a valve is at the corresponding firing instant. In this subsection, when calculating the post-fault extinction angle, it is assumed that the trigger pulse is applied to the valve at the fault time and that the commutating voltage changes instantaneously during the fault, which is the worst case. Therefore, the calculated extinction angle for this criterion is the lower bound of the $\gamma$ values for different fault times.

\section{Spatial-temporal discreteness based approach to CF assessment}

By combining the proposed criterion with results from a short-circuit current calculating program, the assessment of a CF caused by AC system faults can be performed efficiently. The assessment approach is applicable to any fault position and any fault type. The detailed procedures of the proposed assessment approach are summarized as follows:

1) Obtain the pre-fault commutating voltage, the pre-fault ignition advance angle, and the pre-fault extinction angle of the HVDC system to be assessed, i.e., $E_{a c 0}$, $\beta_{0}$, and $\gamma_{0}$.

2) Calculate the post-fault commutating voltage magnitude $E_{a c}$ and the phase shift $\Delta \varphi$ using a short-circuit current calculating program for a given fault position, type and resistance.

3) Substitute these parameters into (15) to obtain the post-fault extinction $\gamma_{\mathrm{f}}$ angle. By comparing $\gamma_{\mathrm{f}}$ with $\gamma_{\text {min }}$, occurrences of CF can be identified. If and only if $\gamma_{\mathrm{f}} \in\left[\gamma_{\min }, \gamma_{0}\right]$ does the commutation succeed.

Using the proposed assessment method, two practical applications in CF assessment can be conducted.

One is to scan the AC system contingencies that cause $\mathrm{CF}$ and to assess the weak area where multi-circuit HVDC systems are prone to concurrent CFs. The critical fault resistance (CFR), with which a fault causes a critical CF in a HVDC system (i.e., $\gamma_{\mathrm{f}} \approx \gamma_{\text {min }}$ ), is usually employed as the index in this assessment case. By scanning fault sets, the CFRs for different fault positions can be obtained and ranked, and then the severity level for different positions of AC faults can be assessed. Thus the fault area likely to cause $\mathrm{CF}$ can be identified. The fault area likely to cause concurrent $\mathrm{CF}$ in multi-infeed HVDC systems is the intersection of the CF-sensitive areas of different HVDC systems.

The other application is to assess the ability of different HVDC systems to avoid CF for a given AC system fault, and to identify the scope for that fault to impact the commutation in a HVDC converter. Likewise, the CFR is employed as the index. By ranking the CFRs of different HVDC systems for a given position of AC fault, this assessment can be achieved.

As shown in the above analysis, the assessment method proposed in this paper does not rely on electromagnetic transient simulations, so it is highly efficient and is applicable to large-scale power systems. Furthermore, because it considers both the magnitude drop and the phase shift of the commutating voltage, the assessment results are more accurate than those obtained with the commonly used 
methods, which consider only the influence of voltage magnitude drop.

\section{Case studies}

\subsection{System introduction}

The China Southern Power Grid (CSG) is known as one of the largest AC/DC hybrid transmission systems in the world. As the receiving-end power grid of CSG, Guangdong provincial grid is characterized by highly clustered load and high proportions of power transmitted from other provincial grids. Especially in the Pearl River Delta grids, the $500 \mathrm{kV}$ networks are in close mutual proximity, and there are multi-infeed HVDC systems: four $\pm 500 \mathrm{kV} \mathrm{DC}$ links and one $\pm 800 \mathrm{kV}$ DC link, namely TG-HVDC, SGHVDC, GZ-HVDC, XA-HVDC, and YG-HVDC. The impacts of a severe fault in the $500 \mathrm{kV}$ grids can be transmitted almost unimpeded to more than one inverter station, thus causing concurrent $\mathrm{CF}$ in inverter stations. If the fault cannot be tripped in time, more than one HVDC system is likely to suffer successive $\mathrm{CF}$, even converter blocking, raising the risk of widespread blackouts. Therefore, establishing a quick and efficient method for $\mathrm{CF}$ assessment is critical.

\subsection{Validation of spatial-temporal discreteness}

In PSCAD/EMTDC models of the CSG, CFRs for AC system faults occurring at different buses and with different fault times can be obtained by adjusting values of fault resistance. To simplify the analysis, without loss of generality, a three-phase fault and its impact on GZ-HVDC is taken as an example. Three-phase balanced faults with different initial fault voltage angles are applied at HD, LD, DG, SX, and SZ $500 \mathrm{kV}$ power stations, and the CFRs and the critical commutation failure voltages (CCFVs) for different initial fault voltage angles (related to the fault times) are plotted in Fig. 6.

The temporal discreteness of $\mathrm{AC}$ faults causing $\mathrm{CF}$ is illustrated in Fig. 6. When a fault is applied at HD station, the most adverse initial fault voltage angles for commutation are $6^{\circ}$ and $36^{\circ}$, and the most favorable angles are $24^{\circ}$ and $54^{\circ}$. Further simulations show that the change cycles of CFRs and CCFVs are $30^{\circ}$.

For faults at different buses, it is observed that CFRs in the ascending order are SZ, DG, SX, HD, and LD stations, regardless of the initial fault voltage angle, and CCFVs in the ascending order are also SZ, DG, SX, HD, and LD stations. In order to facilitate the analysis of spatial discreteness, the average values of CFRs and CCFVs are calculated, as listed in Table 1 with steady-state voltage

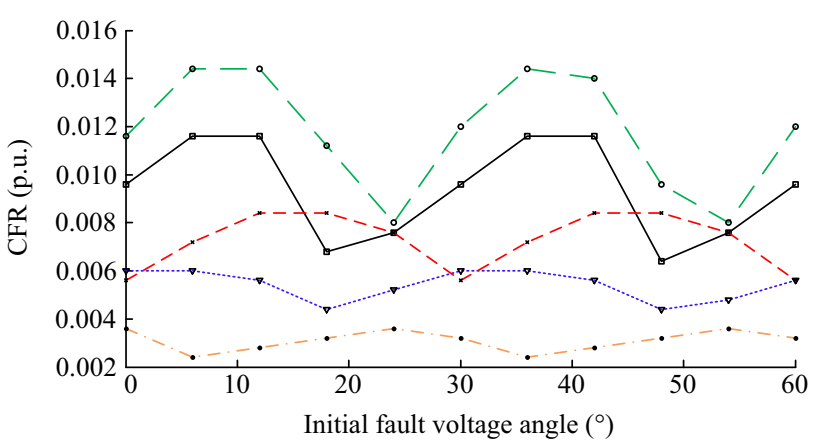

(a) CFRs

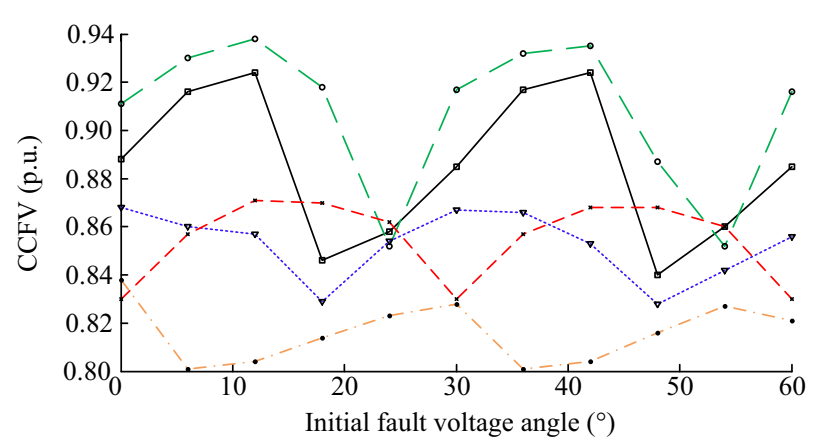

(b) CCFVs

$$
\rightarrow-\mathrm{HD} ; \multimap-\mathrm{LD} ; \cdots \cdots \cdots \cdot \mathrm{DG} ;-*-\mathrm{SX} ;-\bullet \mathrm{SZ}
$$

Fig. 6 Electrical characteristics of critical CF

phases of the stations and electrical proximities of fault stations to the inverter.

Table 1 shows that all steady-state voltage phases of these AC stations lag behind the voltage phase of the commutation bus $\left(29.9^{\circ}\right)$, and the ascending order is SZ, SX, DG, LD, and HD. According to the mutual impedances, electrical proximities of fault positions to the inverter in the descending order are SZ, DG, SX, HD, and $\mathrm{LD}$, which is the ordering of the table.

From Table 1, it is also observed that the ranks of CFRs and CCFVs are consistent with the electrical proximities of fault positions to the inverter, which means that the closer the electrical proximity, the smaller is the CFR and the lower is the CCFV. For this reason, faults occurring at SZ station have less influence on commutation compared with faults occurring at other stations, and faults at LD station are the most unfavorable.

On the other hand, because all steady-state voltage phases of these AC stations lag behind the voltage phase of the commutation bus, all the faults are adverse to $\mathrm{CF}$ as analyzed in Section 2. Among all the stations, SZ has the most lagging steady-state voltage phase and HD station has the least lagging. From the viewpoint of voltage phase, faults occurring at HD station have less influence on commutation compared with faults occurring at other stations, and faults at SZ station are the most unfavorable. 
Table 1 Parameters reflecting spatial discreteness of AC faults

\begin{tabular}{lllll}
\hline Station & Phase $\left({ }^{\circ}\right)$ & Mutual impedance (p.u.) & Average CFR (p.u.) & Average CCFV (p.u.) \\
\hline SZ & 21.1 & 0.000973 & 0.00308 & 0.816 \\
DG & 23.9 & 0.001214 & 0.00540 & 0.853 \\
SX & 23.6 & 0.001379 & 0.00728 & 0.855 \\
HD & 29.4 & 0.001736 & 0.00940 & 0.886 \\
LD & 27.5 & 0.001918 & 0.01180 & 0.908 \\
\hline
\end{tabular}

Combining the effects of the electrical proximity and the steady-state voltage phase, the above simulation results show that the electrical proximity of the fault position to the inverter is the main cause of spatial discreteness of AC faults.

When a three-phase fault and a single-phase fault are applied at BJ station and ZC station, respectively, the lower bounds of CCFVs of different HVDC systems in the CSG for different fault types are shown in Fig. 7. In this figure, the vertical solid line denotes a three-phase fault, and the vertical dotted line denotes a single-phase fault.

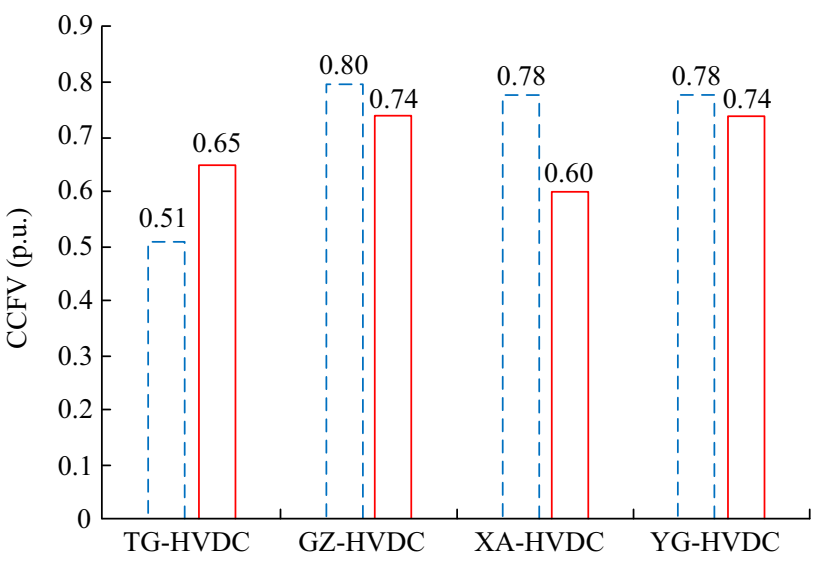

(a) Faults occur at BJ station

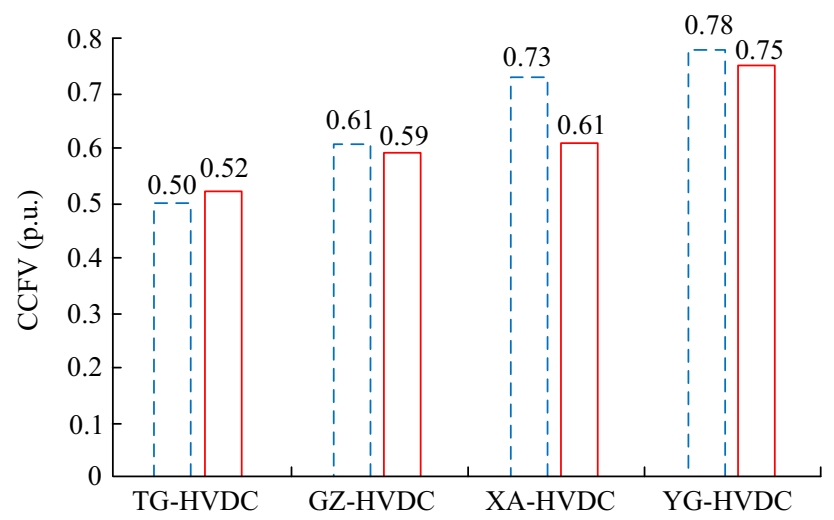

(b) Faults occur at ZC station

¿_ュ Single-phase fault; $\square$ Three-phase fault

Fig. 7 Lower boundary of CCFVs of different HVDC systems
From Fig. 6b, the differences among CCFVs of one HVDC system for different fault positions and fault times are very large, and Fig. 7 shows that the differences among the CCFVs of different HVDC systems for the same fault are also very large. This indicates a large discreteness in the voltage drop condition that causes $\mathrm{CF}$ and means that the maximum permissible voltage drop criterion does not adequately reflect the characteristics of CF. From Fig. 7b, it is noted that for TG-HVDC, a single-phase fault can more easily cause CF than a three-phase fault, in contrast to the common viewpoint that a three-phase fault is more severe and more likely to cause $\mathrm{CF}$.

\subsection{CF assessment in CSG}

Taking GZ-HVDC as an example, assessments of fault areas that cause $\mathrm{CF}$ are carried out by PSCAD/EMTDC simulations and by the proposed method, for comparison. Four types of AC system faults are applied in the PSCAD/ EMTDC models of the CSG: a three-phase fault, a singlephase grounding fault, a two-phase grounding fault, and a two-phase short-circuit fault. The faults are applied at MM, YJ, XH, SX, KP, QY, HY, HX, HZ, and MZ $500 \mathrm{kV}$ power stations, which are far from inverter stations and are likely to be the boundary stations.

The CF assessment results for GZ-HVDC are shown in Tables 2, 3, 4 and 5. The symbol " $x$ " denotes no CF and " $\sqrt{ }$ " means that a CF occurs. For convenient reference, in place of " $x$ ", extinction angles of converter valves are recorded in the assessment results when the proposed method indicates no $\mathrm{CF}$. " $\mathrm{V}_{1} \rightarrow \mathrm{V}_{3}$ " denotes the commutation from $V_{1}$ to $V_{3}$, " $V_{3} \rightarrow V_{5}$ " denotes the commutation from $V_{3}$ to $V_{5}$, and so on. Note that in the assessment results for the proposed method, as long as there is one valve failing to commutate $\left(\gamma_{\mathrm{f}}\right.$ does not fall within the range $\left.\left[\gamma_{\min }, \gamma_{0}\right]\right), \mathrm{CF}$ is considered to occur in the relevant DC system.

In the tables above, the asterisk superscript $*$ denotes that there is a difference between the assessment result of the simulation and that of the proposed method: there is no $\mathrm{CF}$ according to the simulation result, while a $\mathrm{CF}$ is identified by the proposed method. Where there are 
Table 2 CF assessment results of GZ-HVDC for three-phase faults

\begin{tabular}{|c|c|c|c|c|c|c|c|}
\hline \multirow[t]{2}{*}{ AC station } & \multirow[t]{2}{*}{ Simulation results } & \multicolumn{6}{|c|}{ Results of proposed method } \\
\hline & & $\mathrm{V}_{1} \rightarrow \mathrm{V}_{3}$ & $\mathrm{~V}_{3} \rightarrow \mathrm{V}_{5}$ & $\mathrm{~V}_{5} \rightarrow \mathrm{V}_{1}$ & $\mathrm{~V}_{4} \rightarrow \mathrm{V}_{6}$ & $\mathrm{~V}_{6} \rightarrow \mathrm{V}_{2}$ & $\mathrm{~V}_{2} \rightarrow \mathrm{V}_{4}$ \\
\hline MM & $\sqrt{ }$ & $\sqrt{ }$ & $\sqrt{ }$ & $\sqrt{ }$ & $\sqrt{ }$ & $\sqrt{ }$ & $\sqrt{ }$ \\
\hline YJ & $\sqrt{ }$ & $\sqrt{ }$ & $\sqrt{ }$ & $\sqrt{ }$ & $\sqrt{ }$ & $\sqrt{ }$ & $\sqrt{ }$ \\
\hline $\mathrm{XH}$ & $\sqrt{ }$ & $\sqrt{ }$ & $\sqrt{ }$ & $\sqrt{ }$ & $\sqrt{ }$ & $\sqrt{ }$ & $\sqrt{ }$ \\
\hline SX & $\sqrt{ }$ & $\sqrt{ }$ & $\sqrt{ }$ & $\sqrt{ }$ & $\sqrt{ }$ & $\sqrt{ }$ & $\sqrt{ }$ \\
\hline KP & $\sqrt{ }$ & $\sqrt{ }$ & $\sqrt{ }$ & $\sqrt{ }$ & $\sqrt{ }$ & $\sqrt{ }$ & $\sqrt{ }$ \\
\hline QY & $\sqrt{ }$ & $\sqrt{ }$ & $\sqrt{ }$ & $\sqrt{ }$ & $\sqrt{ }$ & $\sqrt{ }$ & $\sqrt{ }$ \\
\hline HY & $\sqrt{ }$ & $\sqrt{ }$ & $\sqrt{ }$ & $\sqrt{ }$ & $\sqrt{ }$ & $\sqrt{ }$ & $\sqrt{ }$ \\
\hline HX & $\sqrt{ }$ & $\sqrt{ }$ & $\sqrt{ }$ & $\sqrt{ }$ & $\sqrt{ }$ & $\sqrt{ }$ & $\sqrt{ }$ \\
\hline $\mathrm{HZ}$ & $\sqrt{ }$ & $\sqrt{ }$ & $\sqrt{ }$ & $\sqrt{ }$ & $\sqrt{ }$ & $\sqrt{ }$ & $\sqrt{ }$ \\
\hline MZ* & $x$ & $\sqrt{ }$ & $\sqrt{ }$ & $\sqrt{ }$ & $\sqrt{ }$ & $\sqrt{ }$ & $\sqrt{ }$ \\
\hline
\end{tabular}

Table $3 \mathrm{CF}$ assessment results of GZ-HVDC for single-phase grounding faults

\begin{tabular}{|c|c|c|c|c|c|c|c|}
\hline \multirow[t]{2}{*}{ AC station } & \multirow[t]{2}{*}{ Simulation results } & \multicolumn{6}{|c|}{ Results of proposed method $\left(^{\circ}\right)$} \\
\hline & & $\mathrm{V}_{1} \rightarrow \mathrm{V}_{3}$ & $\mathrm{~V}_{3} \rightarrow \mathrm{V}_{5}$ & $\mathrm{~V}_{5} \rightarrow \mathrm{V}_{1}$ & $\mathrm{~V}_{4} \rightarrow \mathrm{V}_{6}$ & $\mathrm{~V}_{6} \rightarrow \mathrm{V}_{2}$ & $\mathrm{~V}_{2} \rightarrow \mathrm{V}_{4}$ \\
\hline MM & $\sqrt{ }$ & $\sqrt{ }$ & 13.255 & $\sqrt{ }$ & $\sqrt{ }$ & 12.984 & $\sqrt{ }$ \\
\hline YJ & $\sqrt{ }$ & $\sqrt{ }$ & $\sqrt{ }$ & $\sqrt{ }$ & $\sqrt{ }$ & $\sqrt{ }$ & $\sqrt{ }$ \\
\hline $\mathrm{XH}$ & $\sqrt{ }$ & $\sqrt{ }$ & 9.222 & $\sqrt{ }$ & $\sqrt{ }$ & $\sqrt{ }$ & $\sqrt{ }$ \\
\hline SX & $\sqrt{ }$ & $\sqrt{ }$ & 7.602 & $\sqrt{ }$ & $\sqrt{ }$ & $\sqrt{ }$ & $\sqrt{ }$ \\
\hline $\mathrm{KP}$ & $\sqrt{ }$ & $\sqrt{ }$ & $\sqrt{ }$ & $\sqrt{ }$ & $\sqrt{ }$ & $\sqrt{ }$ & $\sqrt{ }$ \\
\hline $\mathrm{QY}^{*}$ & $x$ & $\sqrt{ }$ & 14.445 & $\sqrt{ }$ & $\sqrt{ }$ & 13.839 & $\sqrt{ }$ \\
\hline $\mathrm{HY}^{*}$ & $x$ & 9.841 & 14.733 & $\sqrt{ }$ & $\sqrt{ }$ & 13.765 & $\sqrt{ }$ \\
\hline $\mathrm{HX}^{*}$ & $x$ & $\sqrt{ }$ & 8.505 & $\sqrt{ }$ & $\sqrt{ }$ & $\sqrt{ }$ & $\sqrt{ }$ \\
\hline $\mathrm{HZ}$ & $\sqrt{ }$ & $\sqrt{ }$ & 10.915 & $\sqrt{ }$ & $\sqrt{ }$ & $\sqrt{ }$ & $\sqrt{ }$ \\
\hline MZ & $x$ & 14.611 & 16.212 & 11.100 & 11.986 & 15.732 & 10.325 \\
\hline
\end{tabular}

Table 4 CF assessment results of GZ-HVDC for two-phase grounding faults

\begin{tabular}{|c|c|c|c|c|c|c|c|}
\hline \multirow[t]{2}{*}{ AC station } & \multirow[t]{2}{*}{ Simulation results } & \multicolumn{6}{|c|}{ Results of proposed method $\left(^{\circ}\right)$} \\
\hline & & $\mathrm{V}_{1} \rightarrow \mathrm{V}_{3}$ & $\mathrm{~V}_{3} \rightarrow \mathrm{V}_{5}$ & $\mathrm{~V}_{5} \rightarrow \mathrm{V}_{1}$ & $\mathrm{~V}_{4} \rightarrow \mathrm{V}_{6}$ & $\mathrm{~V}_{6} \rightarrow \mathrm{V}_{2}$ & $\mathrm{~V}_{2} \rightarrow \mathrm{V}_{4}$ \\
\hline MM & $\sqrt{ }$ & $\sqrt{ }$ & $\sqrt{ }$ & $\sqrt{ }$ & $\sqrt{ }$ & $\sqrt{ }$ & $\sqrt{ }$ \\
\hline YJ & $\sqrt{ }$ & $\sqrt{ }$ & $\sqrt{ }$ & $\sqrt{ }$ & $\sqrt{ }$ & $\sqrt{ }$ & $\sqrt{ }$ \\
\hline $\mathrm{XH}$ & $\sqrt{ }$ & $\sqrt{ }$ & $\sqrt{ }$ & $\sqrt{ }$ & $\sqrt{ }$ & $\sqrt{ }$ & $\sqrt{ }$ \\
\hline SX & $\sqrt{ }$ & $\sqrt{ }$ & $\sqrt{ }$ & $\sqrt{ }$ & $\sqrt{ }$ & $\sqrt{ }$ & $\sqrt{ }$ \\
\hline $\mathrm{KP}$ & $\sqrt{ }$ & $\sqrt{ }$ & $\sqrt{ }$ & $\sqrt{ }$ & $\sqrt{ }$ & $\sqrt{ }$ & $\sqrt{ }$ \\
\hline QY & $\sqrt{ }$ & $\sqrt{ }$ & $\sqrt{ }$ & $\sqrt{ }$ & $\sqrt{ }$ & $\sqrt{ }$ & $\sqrt{ }$ \\
\hline HY & $\sqrt{ }$ & $\sqrt{ }$ & $\sqrt{ }$ & $\sqrt{ }$ & $\sqrt{ }$ & $\sqrt{ }$ & $\sqrt{ }$ \\
\hline HX & $\sqrt{ }$ & $\sqrt{ }$ & $\sqrt{ }$ & $\sqrt{ }$ & $\sqrt{ }$ & $\sqrt{ }$ & $\sqrt{ }$ \\
\hline $\mathrm{HZ}$ & $\sqrt{ }$ & $\sqrt{ }$ & $\sqrt{ }$ & $\sqrt{ }$ & $\sqrt{ }$ & $\sqrt{ }$ & $\sqrt{ }$ \\
\hline $\mathrm{MZ}^{*}$ & $\times$ & 7.601 & $\sqrt{ }$ & 12.721 & $\sqrt{ }$ & $\sqrt{ }$ & 7.624 \\
\hline
\end{tabular}


Table 5 CF assessment results of GZ-HVDC for two-phase short-circuit faults

\begin{tabular}{|c|c|c|c|c|c|c|c|}
\hline \multirow[t]{2}{*}{ AC station } & \multirow[t]{2}{*}{ Simulation results } & \multicolumn{6}{|c|}{ Results of proposed method $\left(^{\circ}\right)$} \\
\hline & & $\mathrm{V}_{1} \rightarrow \mathrm{V}_{3}$ & $\mathrm{~V}_{3} \rightarrow \mathrm{V}_{5}$ & $\mathrm{~V}_{5} \rightarrow \mathrm{V}_{1}$ & $\mathrm{~V}_{4} \rightarrow \mathrm{V}_{6}$ & $\mathrm{~V}_{6} \rightarrow \mathrm{V}_{2}$ & $\mathrm{~V}_{2} \rightarrow \mathrm{V}_{4}$ \\
\hline MM & $\sqrt{ }$ & $\sqrt{ }$ & $\sqrt{ }$ & $\sqrt{ }$ & $\sqrt{ }$ & $\sqrt{ }$ & $\sqrt{ }$ \\
\hline YJ & $\sqrt{ }$ & $\sqrt{ }$ & $\sqrt{ }$ & $\sqrt{ }$ & $\sqrt{ }$ & $\sqrt{ }$ & $\sqrt{ }$ \\
\hline XH & $\sqrt{ }$ & $\sqrt{ }$ & $\sqrt{ }$ & 7.811 & $\sqrt{ }$ & $\sqrt{ }$ & $\sqrt{ }$ \\
\hline SX & $\sqrt{ }$ & $\sqrt{ }$ & $\sqrt{ }$ & 9.902 & $\sqrt{ }$ & $\sqrt{ }$ & $\sqrt{ }$ \\
\hline $\mathrm{KP}$ & $\sqrt{ }$ & $\sqrt{ }$ & $\sqrt{ }$ & $\sqrt{ }$ & $\sqrt{ }$ & $\sqrt{ }$ & $\sqrt{ }$ \\
\hline QY & $\sqrt{ }$ & 15.645 & $\sqrt{ }$ & $\sqrt{ }$ & $\sqrt{ }$ & 16.238 & $\sqrt{ }$ \\
\hline HY & $\sqrt{ }$ & $\sqrt{ }$ & $\sqrt{ }$ & 12.237 & $\sqrt{ }$ & $\sqrt{ }$ & $\sqrt{ }$ \\
\hline HX & $\sqrt{ }$ & $\sqrt{ }$ & $\sqrt{ }$ & $\sqrt{ }$ & $\sqrt{ }$ & $\sqrt{ }$ & $\sqrt{ }$ \\
\hline $\mathrm{HZ}$ & $\sqrt{ }$ & $\sqrt{ }$ & $\sqrt{ }$ & 9.413 & $\sqrt{ }$ & $\sqrt{ }$ & $\sqrt{ }$ \\
\hline MZ* & $\times$ & 10.153 & $\sqrt{ }$ & 15.764 & 10.294 & $\sqrt{ }$ & 12.931 \\
\hline
\end{tabular}

deviations for unbalanced faults, only some converters are suffering $\mathrm{CF}$ and the rest can commutate successfully; for balanced faults, all converters are identified to suffer a $\mathrm{CF}$ by the proposed method. Because the HVDC model in PSCAD/EMTDC is different from that in the proposed assessment method, and temporal discreteness is not considered in PSCAD/EMTDC simulations, the differences between the assessment results are reasonable.

Except for the ten remote AC stations, all HVDC systems located in the Guangdong grid could suffer $\mathrm{CF}$ for bolted faults occurring at AC stations which are closer to inverters.

Based on the above analyses, for all four types of faults, the assessment results of the proposed method are consistent with the simulation results using PSCAD/EMTDC, except for unbalanced faults at very few stations. The assessment results of the proposed method are conservative, which is preferable from the security assessment viewpoint. In addition, compared with electromagnetic transient simulation methods, the proposed method has a much faster computation speed and thus is more practical for engineering use.

\section{Conclusion}

In this paper, on the basis of systematic analysis of the spatial-temporal discreteness of AC system faults causing $\mathrm{CF}$, a novel minimum extinction angle criterion for $\mathrm{CF}$ and an efficient approach to $\mathrm{CF}$ assessment are proposed. The main conclusions of this study are as follows:

1) The fault time affects the maximum commutating voltage-time area applied by the minimum extinction angle, so AC faults causing $\mathrm{CF}$ possess the characteristic of temporal discreteness. The most adverse fault time for commutation is the firing time of converter valves, the anodes of which are connected to the fault phase.

2) The steady-state voltage phase difference and the electrical distance between a fault bus and a commutating bus affect the magnitude and phase of the postfault commutating voltage, leading to the spatial discreteness of AC faults. The closer the electrical proximity, or the bigger the phase lag of an AC station, the more adverse is the fault position to commutation.

3) Regardless of the symmetry of AC faults, the postfault commutating voltage shows both magnitude drops and phase shifts, which differs from the present viewpoint that there is no phase shift for three-phase unbalanced faults.

4) The voltage drop, the phase shift, and the spatialtemporal discreteness of AC system faults are taken into account in the proposed assessment criterion. Therefore, the proposed assessment method can be applied with high accuracy and for practical assessment of $\mathrm{CF}$ caused by all types of faults occurring at any $\mathrm{AC}$ nodes in large-scale AC/DC hybrid systems.

Acknowledgements This work was supported by National Natural Science Foundation of China (No. 51407079).

Open Access This article is distributed under the terms of the Creative Commons Attribution 4.0 International License (http:// creativecommons.org/licenses/by/4.0/), which permits unrestricted use, distribution, and reproduction in any medium, provided you give appropriate credit to the original author(s) and the source, provide a link to the Creative Commons license, and indicate if changes were made.

\section{References}

[1] Rahimi E, Gole AM, Davies JB et al (2011) Commutation failure analysis in multi-infeed HVDC systems. IEEE Trans Power Deliv 26(1):378-384 
[2] Xue Y, Zhang XP, Yang CH (2016) Elimination of commutation failures of LCC HVDC system with controllable capacitors. IEEE Trans Power Syst 31(4):3289-3299

[3] Zhou J, Liu C, Zhao JJ (2016) Simulation analysis of commutation failure in a multi-infeed HVDC system. In: Proceedings of 2016 China international conference on electricity distribution, Xi'an, China, 10 August 2016, 6 pp

[4] Wei ZH, Liu J, Fang WL et al (2016) Commutation failure analysis in single- and multi-infeed HVDC systems. In: Proceedings of 2016 IEEE PES Asia-Pacific power and energy engineering conference, Xi' an, China, 25-28 October 2016, pp 2244-2249

[5] Hong C (2011) Simulation analysis on the commutation failure and power recovery characteristic of an actual DC transmission system. South Power Syst Technol 5(1):1-7

[6] Rahimi E, Filizadeh S, Gole AM (2005) Commutation failure analysis in HVDC systems using advanced multiple-run methods. In: Proceedings of international conference power system transients, Montreal, Canada, 19-23 June 2005, 5 pp

[7] Thio CV, Davies JB, Kent KL (1996) Commutation failures in HVDC transmission systems. IEEE Trans Power Del 11(2):946-957

[8] Li X, Ye C, Wu J et al (2015) Time discreteness of commutation failure in HVDC transmission. In: Proceedings of 2015 IEEE region 10 conference, Macao, China, 1-4 November 2015, 6 pp

[9] Li XH, Xie YS, Dai MS (2014) Analysis of spatial characteristics of HVDC commutation failure in complex power grid. In: Proceedings of 2014 IEEE PES Asia-Pacific power and energy engineering conference (APPEEC), Hong Kong, China, 7-10 December 2014, 4 pp

[10] Rahimi E (2011) Voltage interactions and commutation failure phenomena in multi-infeed HVDC systems. Dissertation, University of Manitoba

[11] Chen XY, Gole AM, Guo CY (2015) A fast calculation method for the local commutation failure immunity indices in singleand multi-infeed HVDC systems. In: Proceedings of the 11th IET international conference on $\mathrm{AC}$ and $\mathrm{DC}$ power transmission, Birmingham, 10-12 February 2015, 6 pp

[12] Rahimi E, Gole AM, Davies JB et al (2006) Commutation failure in single- and multi-infeed HVDC systems. In: Proceedings of the 8th IEE international conference on AC and DC power transmission, London, 28-31 March 2006, pp 182-186
[13] Xiao H, Li Y, Zhu J et al (2015) Efficient approach to quantify commutation failure immunity levels in multi-infeed HVDC systems. IET Gener Transm Distrib 10(4):1032-1038

[14] Yao S, Yong T (2017) Fast evaluation of commutation failure risk in multi-infeed HVDC systems. IEEE Trans Power Syst. https://doi.org/10.1109/TPWRS.2017.2700045

[15] Burr J, Finney S, Booth C (2015) Comparison of different technologies for improving commutation failure immunity index for LCC HVDC in weak AC systems. In: Proceedings of the 11th IET international conference on $\mathrm{AC}$ and $\mathrm{DC}$ power transmission, Birmingham, 10-12 February 2015, 7 pp

[16] Bauman J, Kazerani M (2007) Commutation failure reduction in HVDC systems using adaptive fuzzy logic controller. IEEE Trans Power Syst 22(4):1995-2002

[17] Chen L, Pan H, Deng CH et al (2015) Study on the application of a flux-coupling-type superconducting fault current limiter for decreasing HVDC commutation failure. Can J Electr Comput Eng 38(1):10-19

Huanhuan YANG received his B.S. degree and M.S. degree from South China University of Technology (SCUT), China, in 2011 and 2012, respectively. He is currently a Ph.D. student in School in Electrical Power Engineering of SCUT. His research interests include HVDC, power system stability and control.

Zexiang CAI received the Ph.D. degree from Tsinghua University, China, in 1991. He has been a professor of School of Electrical Power Engineering of SCUT since 1998. His research interests are HVDC, power system protection relay, power system stability and control.

Xiaohua LI received her B.S. and Ph.D. degrees from Huazhong University of Science and Technology in 1997 and 2003, respectively. Now she is a professor in School of Electrical Power Engineering of SCUT. Her research interests are power system protection relay, automation and HVDC.

Chaoyun YU received her B.S. degree and M.S. degree from South China University of Technology (SCUT), China, in 2013 and 2016, respectively. Her research interests are HVDC, power system stability and control. 